\title{
Integrative Effect of Carvedilol and Aerobic Exercise Training Therapies on Improving Cardiac Contractility and Remodeling in Heart Failure Mice
}

\author{
Andréa S. Vanzelli ${ }^{1}$, Alessandra Medeiros ${ }^{2}$, Natale Rolim ${ }^{3}$, Jan B. Bartholomeu', Telma F. Cunha ${ }^{1}$, \\ Luiz G. Bechara ${ }^{1}$, Enéas R. M. Gomes ${ }^{4}$, Katt C. Mattos ${ }^{1}$, Raquel Sirvente ${ }^{5}$, Vera Salemi ${ }^{5}$, Charles Mady ${ }^{5}$, \\ Carlos E. Negrao ${ }^{1,5}$, Silvia Guatimosim ${ }^{4}$, Patricia C. Brum ${ }^{1 *}$ \\ 1 School of Physical Education and Sport, University of São Paulo, São Paulo, Brazil, 2 Biosciences Department- Federal University of São Paulo, Santos, Brazil, \\ 3 Department of Circulation and Medical Imaging and K.G. Jebsen Center of Exercise in Medicine, Trondheim, Norway, 4 Physiology and Biophysics Department, Federal \\ University of Minas Gerais, Belo Horizonte, Brazil, $\mathbf{5}$ Heart Institute (InCor), University of São Paulo, Medical School, São Paulo, Brazil
}

\begin{abstract}
The use of $\beta$-blockers is mandatory for counteracting heart failure (HF)-induced chronic sympathetic hyperactivity, cardiac dysfunction and remodeling. Importantly, aerobic exercise training, an efficient nonpharmacological therapy to HF, also counteracts sympathetic hyperactivity in HF and improves exercise tolerance and cardiac contractility; the latter associated with changes in cardiac $\mathrm{Ca}^{2+}$ handling. This study was undertaken to test whether combined $\beta$-blocker and aerobic exercise training would integrate the beneficial effects of isolated therapies on cardiac structure, contractility and cardiomyocyte $\mathrm{Ca}^{2+}$ handling in a genetic model of sympathetic hyperactivity-induced $\mathrm{HF}\left(\alpha_{2 \mathrm{~A}} / \alpha_{2 C^{-}}\right.$adrenergic receptor knockout mice, $\mathrm{KO}$ ). We used a cohort of 5-7 mo male wild-type (WT) and congenic mice (KO) with C57Bl6/J genetic background randomly assigned into 5 groups: control (WT), saline-treated KO (KOS), exercise trained KO (KOT), carvedilol-treated KO (KOC) and, combined carvedilol-treated and exercise-trained KO (KOCT). Isolated and combined therapies reduced mortality compared with KOS mice. Both KOT and KOCT groups had increased exercise tolerance, while groups receiving carvedilol had increased left ventricular fractional shortening and reduced cardiac collagen volume fraction compared with KOS group. Cellular data confirmed that cardiomyocytes from KOS mice displayed abnormal $\mathrm{Ca}^{2+}$ handling. KOT group had increased intracellular peak of $\mathrm{Ca}^{2+}$ transient and reduced diastolic $\mathrm{Ca}^{2+}$ decay compared with $\mathrm{KOS}$ group, while $\mathrm{KOC}$ had increased $\mathrm{Ca}^{2+}$ decay compared with KOS group. Notably, combined therapies re-established cardiomyocyte $\mathrm{Ca}^{2+}$ transient paralleled by increased SERCA2 expression and SERCA2:PLN ratio toward WT levels. Aerobic exercise trained increased the phosphorylation of PLN at Ser ${ }^{16}$ and $\mathrm{Thr}^{17}$ residues in both KOT and KOCT groups, but carvedilol treatment reduced lipid peroxidation in KOC and KOCT groups compared with KOS group. The present findings provide evidence that the combination of carvedilol and aerobic exercise training therapies lead to a better integrative outcome than carvedilol or exercise training used in isolation.
\end{abstract}

Citation: Vanzelli AS, Medeiros A, Rolim N, Bartholomeu JB, Cunha TF, et al. (2013) Integrative Effect of Carvedilol and Aerobic Exercise Training Therapies on Improving Cardiac Contractility and Remodeling in Heart Failure Mice. PLoS ONE 8(5): e62452. doi:10.1371/journal.pone.0062452

Editor: Vincenzo Lionetti, Scuola Superiore Sant'Anna, Italy

Received December 12, 2012; Accepted March 21, 2013; Published May 1, 2013

Copyright: (c) 2013 Vanzelli et al. This is an open-access article distributed under the terms of the Creative Commons Attribution License, which permits unrestricted use, distribution, and reproduction in any medium, provided the original author and source are credited.

Funding: Fundação de Amparo a Pesquisa do Estado de São Paulo, São Paulo. SP (FAPESP: 2010/50048-1), FAPEMIG and Instituto Milenio (S.G.), Conselho Nacional de Pesquisa e Desenvolvimento (CNPq, S.G. and P.C.B.) for funding the present investigation, and Laboratories Baldacci S.A. for donating carvedilol. A.S.V. held a scholarship from FAPESP (06/56123-0). P.C.B. holds a scholarship from CNPq (302201/2011-4). The funders had no role in study design, data collection and analysis, decision to publish, or preparation of the manuscript.

Competing Interests: The authors have declared that no competing interests exist.

*E-mail: pcbrum@usp.br

\section{Introduction}

Heart failure (HF) is a common endpoint for many forms of cardiovascular disease. In addition, this syndrome is the leading cause of morbidity and mortality in older individuals [1]. The development of end-stage HF often involves an initial insult to the myocardium that reduces cardiac output and arterial baroreceptor stimulation leading to a compensatory increase in sympathetic nervous system activity, which ultimately results in cardiac dysfunction and remodeling [2,3] In fact, sympathetic hyperactivity is associated with poor prognosis and constitutes an independent predictor of mortality [4,5]. For counteracting sympathetic hyperactivity, the use of $\beta$-blockers is mandatory for HF therapy [6].
The treatment with $\beta$-blockers decreases sympathetic activity measured directly by microneurography performed on the anterior fibular nerve of HF patients [7], and promotes positive impact on cardiac function associated with a reverse remodeling [8]. In a genetic model of $\mathrm{HF}$ based on disruption of $\alpha_{2 \mathrm{~A}} / \alpha_{2 \mathrm{C}^{-}}$ adrenergic receptors from mouse genome, we have previously observed that the third generation $\beta$-blocker, carvedilol, has no impact on exercise capacity but display an anti-cardiac remodeling effect and improves cardiac contractility $[9,10]$, which is independent of changes in isolated cardiac myocyte $\mathrm{Ca}^{2+}$ transients [9]. Accumulated evidence shows that aerobic exercise training is also an important strategy for the prevention and treatment of cardiovascular diseases [11], besides being an efficient adjuvant 
therapy for HF. Aerobic exercise training improves exercise tolerance and cardiac contractility; the later associated with changes in cardiac $\mathrm{Ca}^{2+}$ handling [12-14].

To increase the knowledge about different impact of $\beta$-blockers and aerobic exercise training on cardiac and skeletal muscle, we previously compared the isolated effects of exercise training and carvedilol treatment on exercise tolerance and cardiac contractility and remodeling in mice with an early stage $\mathrm{HF}$ induced by sympathetic hyperactivity $[13,15]$. We observed that both aerobic exercise training and carvedilol therapy improved, to the same extent, the ventricular function in mild HF. However, while the benefits of aerobic exercise training were mainly associated with increased aerobic capacity and capillary density of skeletal muscle, the benefits of carvedilol were restricted to the effect on cardiac structure [15]. Although both carvedilol and aerobic exercise training have been highly recommended to the treatment of $\mathrm{HF}$, it is unknown whether the combination of aerobic exercise training and carvedilol has integrative effects on the treatment of HF. In addition, the cellular basis of associative therapy on cardiac contractility has not been clarified yet.

In the present study, we used a genetic model of sympathetic hyperactivity-induced $\mathrm{HF}$ in mice to determine the combined effects of carvedilol and aerobic exercise training on cardiac structure and function, and overall functional capacity. Furthermore, we studied the expression of proteins involved in cardiac intracellular $\mathrm{Ca}^{2+}$ regulation and $\mathrm{Ca}^{2+}$ transients isolated from cardiomyocytes of all mice studied.

\section{Materials and Methods}

\section{Animal Care}

A cohort of male wild-type (WT) and congenic $\alpha_{2 \mathrm{~A}} / \alpha_{2 \mathrm{C}} \mathrm{ARKO}$ mice $(\mathrm{KO})$ with $\mathrm{C} 57 \mathrm{Bl6} / \mathrm{J}$ genetic background aged 5-7 months was studied. At this age, KO mice display advanced stage cardiomyopathy as previously described [16]. Genotypes were determined by PCR on genomic DNA obtained from tail biopsies using primers to detect the intact and disrupted genes. Mice were maintained in a light-controlled (12-hour light/dark cycle) and temperature-controlled $\left(22^{\circ} \mathrm{C}\right)$ environment and were fed a pellet rodent diet (Nuvital Nutrientes S/A, Curitiba, PR Brazil) ad libitum and had free access to water. Mice were randomly assigned into five groups: control (WT), HF placebo (KOS), HF exercise trained (KOT), HF carvedilol-treated (KOG) and, HF exercise trained and carvedilol-treated (KOCT). This study was carried out in accordance with Ethical Principles of animal research adopted by the Brazilian College of Animal Experimentation (www.cobea. org.br). In addition, this study was approved by the Faculty of Medicine of University of São Paulo Ethics Committee (CEP 897/ 06). The experimental design is shown in Figure 1.

\section{Measurements and Procedures}

Drug treatment and exercise training protocol. Drug treatment consisted of 8 weeks of placebo (saline) or carvedilol (38 mg/kg, Baldacci S.A., SP, Brazil) by gavage. Carvedilol is a third generation non-selective $\beta$-blocker with $\alpha_{1}$-blocking properties with a wide use in HF pharmacological therapy. Carvedilol did not change cardiac function, structure, $\mathrm{Ca}^{2+}$ or cardiac oxidative index in control mice. Therefore, we used only one WT group for further comparisons with KO groups. The dose of carvedilol was optimized to achieve comparable heart rate (HR) levels observed in age matched control group.

Moderate intensity aerobic exercise training was performed on a motor treadmill over 8 weeks, 5 days/week. The running speed and duration of exercise were progressively increased to elicit $60 \%$ of maximal speed, achieved during a graded treadmill exercise protocol, for $60 \mathrm{~min}$. At the fourth week of exercise training, graded exercise test were repeated for readjusting the running speed. This intensity was maintained during the rest of the training period. All untrained mice were exposed to treadmill exercise (5 min) three times a week to become accustomed to exercise protocol and handling. The training sessions were performed during the dark cycle of the mice.

Graded treadmill exercise test. Exercise capacity, estimated by total distance run, was evaluated using a graded treadmill exercise protocol for mice. After being adapted to treadmill exercises over 1 week (10 min of exercise session), mice were placed on the treadmill streak and allowed to acclimatize for at least $30 \mathrm{~min}$. Exercise began at $6 \mathrm{~m} / \mathrm{min}$ with no grade and increased by $3 \mathrm{~m} / \mathrm{min}$ every $3 \mathrm{~min}$ thereafter until exhaustion. The graded treadmill exercise test was performed in WT and $\mathrm{KO}$ mice before and after the aerobic exercise training period. Additionally, it was repeated at the fourth week of exercise training in order to adjust the training intensity as described above.

Cardiovascular measurements. HR and blood pressure were determined noninvasively using a computerized tail-cuff system (BP 2000 Visitech Systems, USA) described elsewhere [17]. Mice were acclimatized to the apparatus during daily sessions over 6 days, 1 week before starting the experimental period. HR measurements were obtained serially in WT and KO mice once a week throughout the 8 weeks of experiments. Noninvasive cardiac function was assessed by two-dimensional guided M-mode echocardiography, in halothane-anesthetized WT and HF mice (Fig. 1). Briefly, mice were positioned in the supine position with front paws wide open, and an ultrasound transmission gel was applied to the precordium. Transthoracic echocardiography was performed using a Sequoia 512 echocardiography machine (Acuson, Mountain View, CA, USA) equipped with a $14-\mathrm{MHz}$ linear transducer and heart rate was kept similar in all groups studied during the evaluation to avoid artifactual changes in fractional shortening. Left ventricle systolic function was estimated by fractional shortening as follows:

Fractional shortening $(\%)=[($ LVEDD - LVESD $) /$ LVEDD $] \times$ 100, where LVEDD means left ventricular end-diastolic dimension, and LVESD means left ventricular end-systolic dimension.

For cardiac structural analysis, at the end of intervention period (Fig. 1), the heart was stopped at diastole (KCl, $14 \mathrm{mM}$ ) and dissected to obtain the left ventricle, which corresponds to the remaining organ upon removal of both atria and free wall of the right ventricle. For morphometric analysis, left ventricle samples obtained from the free wall, at the level of papillary muscle, were fixed in $4 \%$ buffered formalin and embedded in paraffin, cut in $4 \mu \mathrm{m}$ sections and subsequently stained with hematoxylin and eosin. Two randomly selected sections from each animal were visualized by light microscopy using an objective with a calibrated magnification $(400 \times)$. Myocytes with visible nucleus and intact cellular membranes were chosen for diameter determination. The width of individually isolated cardiomyocyte displayed on a viewing screen was manually traced, across the middle of the nuclei, with a digitizing pad and determined by a computer assisted image analysis system (Quantimet 520; Cambridge Instruments, UK). For each animal approximately 15 visual fields were analyzed. Quantification of left ventricular fibrosis was achieved by Sirius red staining. Two randomly selected sections from each animal were visualized by light microscopy using an objective with a calibrated magnification of $200 \times$. Interstitial collagen area was quantified by a computer assisted image analysis system 


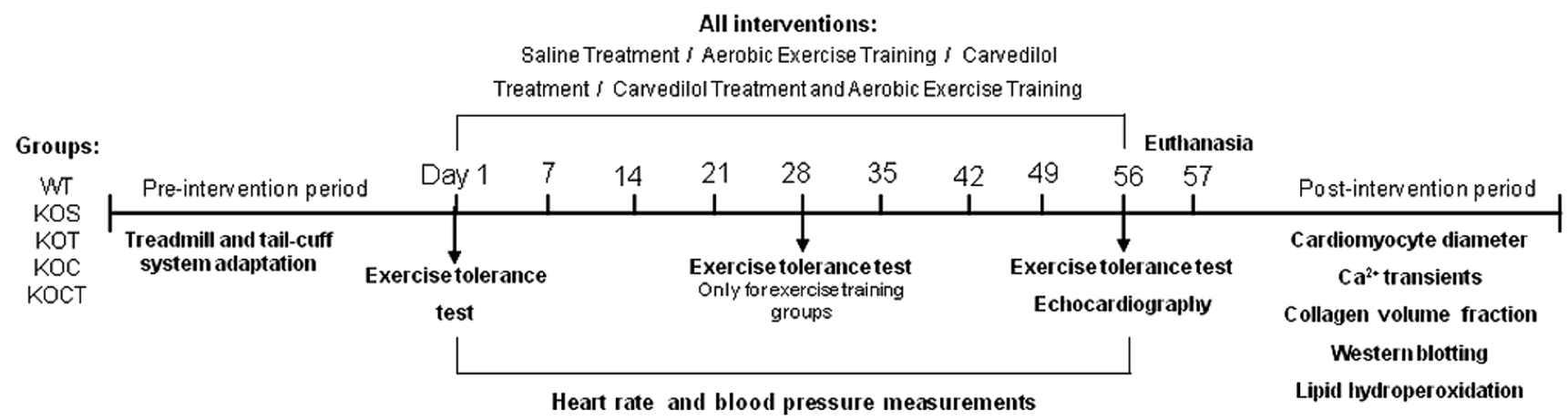

Figure 1. Experimental design. WT, wild type mice (control group); KOS, heart failure placebo; KOT, heart failure exercise trained; KOC, heart failure carvedilol-treated; and, KOCT, heart failure carvedilol-treated and exercise trained mice. doi:10.1371/journal.pone.0062452.g001

(Quantimet 520; Cambridge Instruments, UK). For each animal approximately five visual fields were analyzed.

Gardiomyocyte isolation and $\mathbf{C a}^{2+}$ recording. To verify the cardiomyocyte $\mathrm{Ca}^{2+}$ transients, the other part of the animals received an intraperitoneal injection of pentobarbital sodium $(100 \mathrm{mg} / \mathrm{kg})$, and after full anesthesia the heart was rapidly removed. Cardiac ventricular myocytes were isolated, and imaged for $\left[\mathrm{Ca}^{2+}\right] \mathrm{i}$ as previously described [18]. The $\mathrm{Ca}^{2+}$ level measured with confocal microscopy was reported as $\mathrm{F} / \mathrm{F} 0$, where $\mathrm{F} 0$ is the resting $\mathrm{Ca}^{2+}$ fluorescence. Images were obtained using the ZEISS Meta confocal microscope from CEMEL (Biological Sciences Institute, UFMG, Brazil).

Antibodies. Mouse monoclonal antibodies to SERCA2 (1:2,500), Phospholamban (PLN, 1:500) and $\mathrm{Na}^{+}-\mathrm{Ca}^{2+}$ exchanger (NCX, 1:2,000) were obtained from Affinity BioReagents (Golden, $\mathrm{CO}, \mathrm{USA})$; rabbit polyclonal antibody to protein phosphatase type 1 (PP1, 1:1,000) were obtained from Upstate (Lake Placid, NY, USA); phospho-Ser ${ }^{16}$-PLN $(1: 5,000)$ and phospho-Thr ${ }^{17}$-PLN $(1: 5,000)$ by Badrilla (Leeds, UK); Ryanodine antibody (RyR) was obtained from ABR Incorporation, EUA (1:5,000); Glyceraldehyde-3-phosphate dehydrogenase (GAPDH, 1:2,000) was obtained from Advanced Immunochemical (Long Beach, CA, USA). Targeted bands were normalized to cardiac GAPDH.

Western blot analysis. Left ventricular homogenates were analyzed by Western blotting to compare SERCA2, PLN, phospho-Ser ${ }^{16}$-PLN, phospho-Thr ${ }^{17}$-PLN, NCX, PP1 and RyR. Briefly, liquid nitrogen-frozen ventricles isolated from WT and HF mice were homogenized in a buffer containing $50 \mathrm{mM}$ potassium phosphate buffer ( $\mathrm{pH} 7.0), 0.3 \mathrm{M}$ sucrose, $0.5 \mathrm{mM}$ DTT, $1 \mathrm{mM}$ EDTA (pH 8.0), $0.3 \mathrm{mM}$ PMSF, $10 \mathrm{mM} \mathrm{NaF}$, and phosphatase inhibitor cocktail (1:100, Sigma-Aldrich; Saint Louis, MO). Samples were subjected to SDS-PAGE in polyacrylamide gels (6\% or $10 \%$ depending on protein molecular weight). After electrophoresis, proteins were electrotransferred to nitrocellulose membrane (Amersham Biosciences; Piscataway, NJ, USA). Equal loading of samples $(50 \mathrm{mg})$ and even transfer efficiency were monitored with the use of $0.5 \%$ Ponceau S staining of the blot membrane. The blotted membrane was then blocked ( $5 \%$ nonfat dry milk, $10 \mathrm{mM}$ Tris-HCl, pH 7.6, $150 \mathrm{mM} \mathrm{NaCl}$, and $0.1 \%$ Tween 20) for $2 \mathrm{~h}$ at room temperature and incubated with specific antibodies overnight at $4^{\circ} \mathrm{C}$. Binding of the primary antibody was detected with the use of peroxidase-conjugated secondary antibodies (rabbit or mouse depending on the protein, 1:10,000, for $1 \mathrm{~h}: 30 \mathrm{~min}$ at room temperature) and developed using enhanced chemiluminescence (Amersham Biosciences, USA) detected by autoradiography. Quantification analysis of blots was performed with the use of Scion Image software (Scion based on NIH image).

Lipid hydroperoxides. Myocardial lipid hydroperoxide measurement was evaluated as an index of cardiac oxidative injury by the ferrous oxidation-xylenol orange technique (FOX2) [19]. Heart samples were homogenized $(1: 20 \mathrm{w} / \mathrm{v})$ in cold phosphate-buffered saline $(100 \mathrm{mM}, \mathrm{pH} 7.4)$ and immediately centrifuged at $12,000 \mathrm{~g}$ for $20 \mathrm{~min}$ at $4^{\circ} \mathrm{C}$.

Proteins were precipitated with trichloroacetic acid $(10 \% \mathrm{w} / \mathrm{v})$ and supernatant was mixed with FOX reagent and incubated for $30 \mathrm{~min}$. The absorbance of the sample was read at $560 \mathrm{~nm}$.

\section{Statistical Analysis}

All variables showed normal distribution, when analyzed using the Shapiro-Wilk normality test, and therefore, the parametric statistical analysis was used. Data were expressed as mean \pm standard error. The variables (peak of $\mathrm{Ca}^{2+}$ transient, decay $\mathrm{Ca}^{2+}$ transient, fractional shortening, cardiomyocyte diameter, collagen fraction and western blot analysis) were compared among groups by one-way analysis of variance (ANOVA) or one way ANOVA with repeated measures (exercise tolerance, heart rate, and blood pressure). For mortality rate, log rank analysis was used (GehanBreslow-Wilcoxon Test). In case of statistical significance, Tukey's post hoc test was adopted. For all analyses, we adopted the significance level of $\mathrm{P}<0.05$. The software used for statistical analysis was Statistica version 7.0.

\section{Results}

\section{Effects of Therapies in Heart Rate and Blood Pressure}

KOS displayed baseline tachycardia when compared with agematched control mice even though resting blood pressure was similar among all groups $(\mathrm{P}<0.05$, Figures $2 \mathrm{~A}$ and $2 \mathrm{~B})$. Blood pressure remained unchanged while baseline $\mathrm{HR}$ was reduced in WT group levels from the fourth week in both isolated and combined carvedilol and aerobic exercise training therapies, showing similar effectiveness of the therapies to reduce $\mathrm{HR}$ in KO toward WT levels $(\mathrm{P}<0.05$, Figures $2 \mathrm{~A}$ and $2 \mathrm{~B})$.

\section{Effects of Therapies on Survival, Exercise Tolerance, and Cardiac Function}

While KOS mice presented $40 \%$ mortality rate after eight weeks of the study, aerobic exercise training, carvedilol treatment or carvedilol associated with exercise training significantly reduced HF mice mortality to $20 \%, 19 \%$ and $13 \%$, respectively $(\mathrm{P}<0.05$, Figure 3A). Exercise tolerance was reduced in KOS after 8 weeks 
A

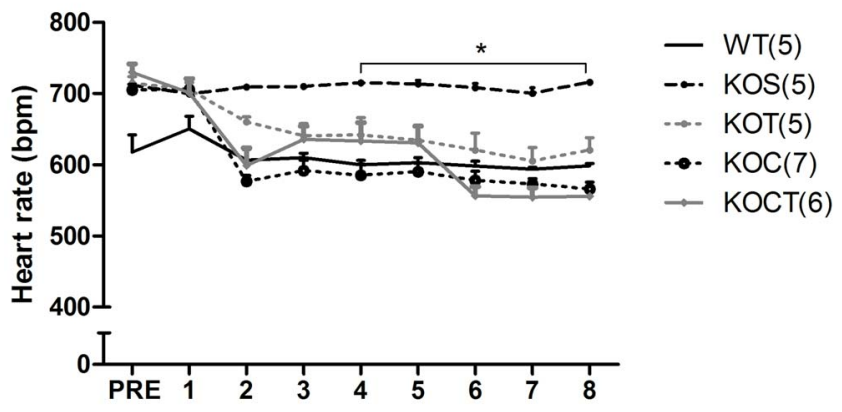

B

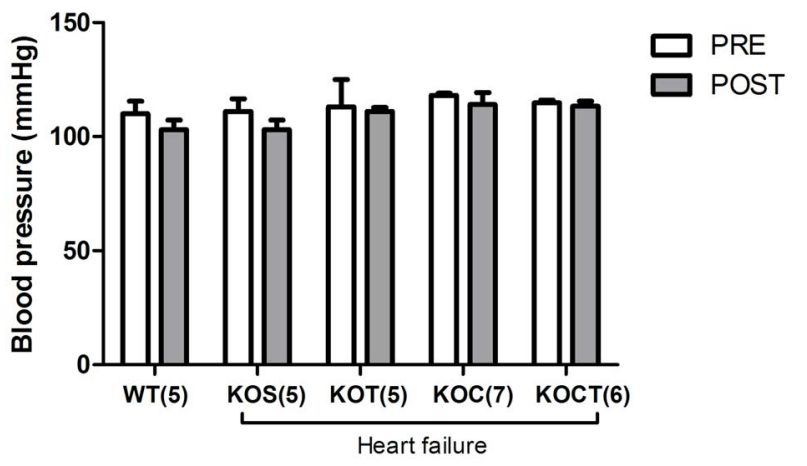

Figure 2. Heart rate (A) and blood pressure (B) during interventions in control (WT), heart failure placebo (KOS), heart failure exercise trained (KOT), heart failure carvediloltreated (KOC) and, heart failure carvedilol-treated and exercise-trained (KOCT) mice. Note that all interventions decreased to the same extent the baseline HR of KO mice, which became similar to HR of WT group. Data are presented as mean \pm SE. The number of animals studied is shown between parentheses (Panel A) or indicated by numerals on the abscissa (Panel $B$ ). ${ }^{*} \mathrm{P}<0.05$ vs. other groups (groups indicated by lines).

doi:10.1371/journal.pone.0062452.g002

of the study $(\mathrm{P}<0.05$, Figure $3 \mathrm{~B})$. Both isolated aerobic exercise training and combined aerobic exercise training and carvedilol increased exercise tolerance in $\mathrm{KO}$ mice $(\mathrm{P}<0.05$, Figure $3 \mathrm{~B})$ and exercise performance was comparable to that achieved for WT trained mice (data not shown). As expected, fractional shortening was reduced in KOS mice, and both carvedilol treatment and carvedilol combined to aerobic exercise training increased fractional shortening to WT mice levels $(\mathrm{P}<0.05$, Figure $3 \mathrm{C})$. The result cannot be explained by differences within groups in HR, because there were no significant differences between the HR under anesthesia $(474 \pm 11.8,475 \pm 7.6,471 \pm 16.5,465 \pm 20$, $467 \pm 18.8 \mathrm{bpm}$ for WT, KOS, KOT, KOC and KOCT, respectively). Therefore, HR would not be expected to artifactually change the fractional shortening.

\section{Effects of Therapies in Cardiac Structure, Renal Mass and Lung Water Content}

As expected, KOS mice displayed increased left ventricular mass $(\mathrm{P}<0.05$, Table 1), cardiomyocyte cross sectional diameter and ventricular collagen volume fraction $(\mathrm{P}<0.05$, Figure 4$)$ compared with WT mice suggesting cardiac remodeling. All isolated and combined therapies were equally efficient in reducing left ventricular mass $(\mathrm{P}<0.05$, Table 1$)$ and cardiomyocyte cross sectional area in KOS mice to levels comparable to WT mice
A

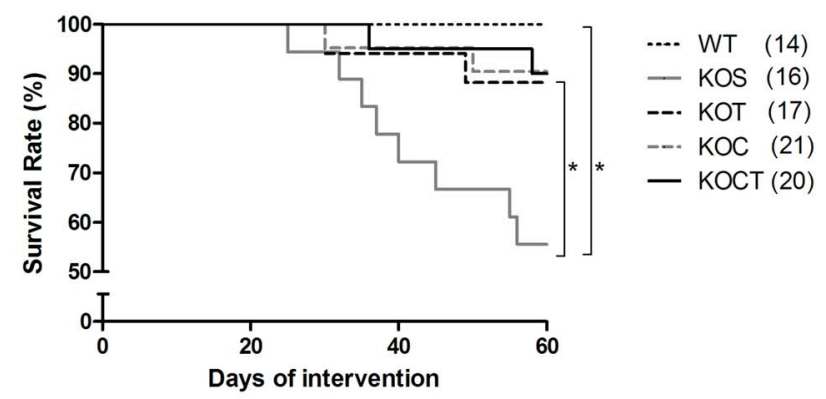

B

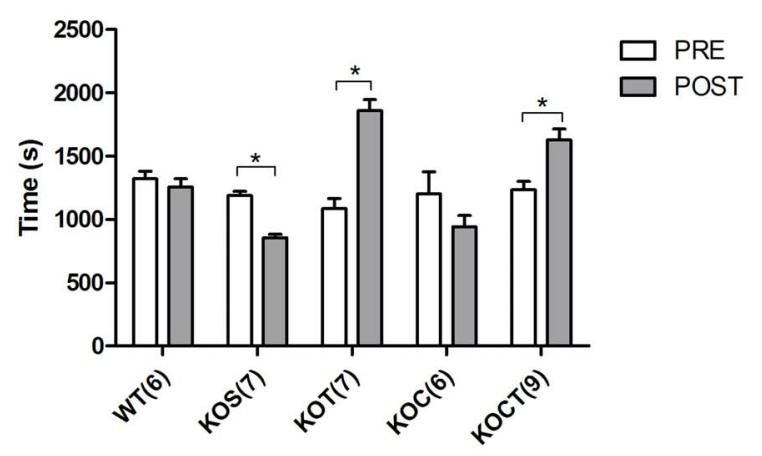

C

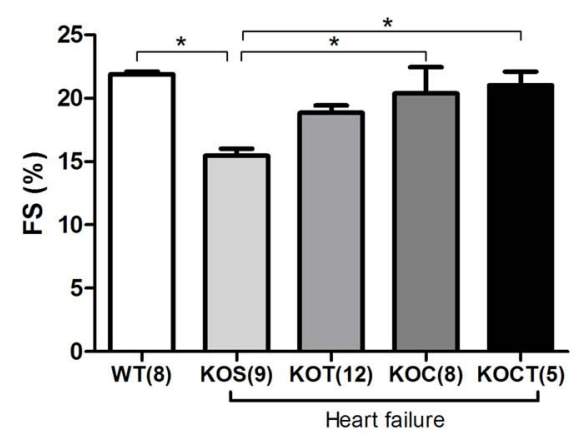

Figure 3. Survival (A), Exercise tolerance (B) and fractional shortening (FS, C) in control (WT), heart failure placebo (KOS), heart failure exercise trained (KOT), heart failure carvediloltreated $(K O C)$ and, heart failure carvedilol-treated and exercise trained (KOCT) mice. FS was evaluated after 8 weeks of intervention. Note that all interventions reduced mortality rate. However, only trained groups (KOT and KOCT) increased exercise tolerance. Data are presented as mean $\pm \mathrm{SE}$. The number of animals studied is shown between parentheses (Panel A) or indicated by numerals on the abscissa (Panels B and C). Panel A: ${ }^{*} P<0.05$ between KOS and WT, and among KOS and other groups. Panel $B$ and $C:{ }^{*} P<0.05$ among groups indicated by lines.

doi:10.1371/journal.pone.0062452.g003

$(\mathrm{P}<0.05$, Figure 4A). However, only carvedilol treatment and carvedilol combined to aerobic exercise training reduced the left ventricle collagen volume fraction to WT levels $(\mathrm{P}<0.05$, Figure 4B).

KOS mice displayed increased kidney mass compared with WT mice $(\mathrm{P}<0.05$, Table 1$)$ with no significant changes in plasma creatinine (data not shown). No differences were observed in lung mass and wet:dry lung mass between KOS and WT mice (Table 1). 
Table 1. Body mass, cardiac chambers mass, kidney mass, lung mass and wet: dry lung mass ratio of control (WT), heart failure placebo (KOS), heart failure exercise trained (KOT), heart failure carvedilol-treated (KOC) and, heart failure carvedilol-treated and exercise-trained (KOCT).

\begin{tabular}{|c|c|c|c|c|c|c|}
\hline & Body mass (g) & $\begin{array}{l}\text { Right ventricle } \\
\text { mass (g) }\end{array}$ & Left ventricle mass (g) & Kidney mass (g) & Lung mass (g) & $\begin{array}{l}\text { Wet :dry lung } \\
\text { mass (g) }\end{array}$ \\
\hline WT & $28 \pm 0.9(10)$ & $0.03 \pm 0.01$ & $0.09 \pm 0.041(10)$ & $0.30 \pm 0.01(6)$ & $0.20 \pm 0.01$ (9) & $4.85 \pm 0.53(8)$ \\
\hline KOS & $29 \pm 0.8(7)$ & $0.03 \pm 0.00(7)$ & $0.11 \pm 0.011 *(7)$ & $0.37 \pm 0.01 *(4)$ & $0.20 \pm 0.02(7)$ & $6.18 \pm 0.67(7)$ \\
\hline KOT & $29 \pm 1.3(5)$ & $0.03 \pm 0.001$ & $0.09 \pm 0.001^{\#}(4)$ & $0.37 \pm 0.01 *(4)$ & $0.17 \pm 0.01(5)$ & $4.89 \pm 0.11(5)$ \\
\hline KOC & $27 \pm 0.8(8)$ & $0.03 \pm 0.01(8)$ & $0.09 \pm 0.002^{\#}(8)$ & $0.32 \pm 0.02^{\# \ddagger}(4)$ & $0.19 \pm 0.01(5)$ & $4.85 \pm 0.21(5)$ \\
\hline КОСТ & $27 \pm 0.7(12)$ & $0.03 \pm 0.001(12)$ & $0.09 \pm 0.041^{\#}(12)$ & $0.32 \pm 0.01^{\# \ddagger}(12)$ & $0.26 \pm 0.02(12)$ & $4.76 \pm 0.20(11)^{\#}$ \\
\hline
\end{tabular}

${ }^{*} \mathrm{P}<0.05$ vs. WT;

${ }^{\#} \mathrm{P}<0.05$ vs. KOS;

${ }_{\mathrm{F}} \mathrm{P}<0.05$ vs. KOT. The number of animals studied is shown between parentheses.

doi:10.1371/journal.pone.0062452.t001

Combined carvedilol and aerobic exercise training, but not isolated therapies, significantly reduced kidney mass and wet:dry lung mass ratio when compared to $\operatorname{KOS}$ group $(\mathrm{P}<0.05$, Table 1$)$.

\section{Effects of Therapies in Cardiomyocyte $\mathrm{Ca}^{2+}$ Transients and Expression of Cardiac $\mathrm{Ca}^{2+}$ Handling Proteins}

Given the fact that $\mathrm{Ca}^{2+}$ handling is closely linked to cardiac contractile function regulation, we further examined the $\mathrm{Ca}^{2+}$ transients in isolated cardiomyocytes from all groups studied. Cardiomyocytes from KOS mice displayed reduced peak of $\mathrm{Ca}^{2+}$ transient compared with WT mice $(\mathrm{P}<0.05$, Figure $5 \mathrm{~A})$. Aerobic exercise training increased peak of $\mathrm{Ca}^{2+}$ transient compared with cardiomyocytes from KOS mice $(\mathrm{P}<0.05$, Figure 5A), which was not changed by isolated carvedilol treatment (Figure 5A). Surprisingly, carvedilol associated with aerobic exercise training had no impact on peak of $\mathrm{Ca}^{2+}$ transient (Figure 5A). Diastolic $\mathrm{Ca}^{2+}$ decay were not changed in KOS group when compared to WT group (Figure 5B), but increased by isolated carvedilol therapy $(\mathrm{P}<0.05$, Figure $5 \mathrm{~B})$. Interestingly, both isolated aerobic exercise training and combined carvedilol and aerobic exercise training therapies decreased $\mathrm{Ca}^{2+}$ decay compared with $\mathrm{KOS}$ group $(\mathrm{P}<0.05$, Figure 5B). To gain further insight into this response, we investigated the expression of key cardiac $\mathrm{Ca}^{2+}$ handling proteins. We observed that SERCA2 expression levels were not changed in KOS when compared to WT mice (Figure 6A). Interestingly, combined carvedilol and aerobic exercise training therapies significantly increased SERCA2 expression and SERCA2:PLN ratio compared with WT and KOS mice $(\mathrm{P}<0.05$, Figures $5 \mathrm{~A}$ and $5 \mathrm{~B})$. Phospho-Ser ${ }^{16}$-PLN:PLN ratio was increased by both aerobic exercise training or combined carvedilol and aerobic exercise training therapies in $\mathrm{KO}$ mice compared with WT and KOC, respectively $(\mathrm{P}<0.05$, Figure $6 \mathrm{C})$. The PP1 expression, which is mainly involved in dephosphorylating PLN at $\operatorname{Ser}^{16}$ residue, was similar among groups studied (Figure 6D). Phospho-Thr ${ }^{17}$-PLN:PLN ratio was significantly increased in KOS, KOT and KOCT compared with WT mice $(\mathrm{P}<0.05$, Figure $6 \mathrm{E})$. The KOG group presented similar phospho-Thr ${ }^{17}$-PLN:PLN levels to WT mice (Figure $6 \mathrm{E}$ ).

No changes were observed in NCX, PLN and RyR protein expression among the groups studied (Figures $6 \mathrm{~F}$ and $6 \mathrm{G}$ ). GAPDH protein levels remained unchanged in all blots analyzed and among the four groups studied (data not shown) and were used to normalize the cardiac $\mathrm{Ca}^{2+}$ handling protein levels.

\section{Effects of Therapies on Marker of Oxidative Stress}

As expected, KOS mice displayed significantly increased cardiac lipid peroxides compared with WT mice $(\mathrm{P}<0.05$, Figure 7). Carvedilol treatment or combined carvedilol and aerobic exercise training therapies reduced lipid peroxidation compared with KOS group $(\mathrm{P}<0.05$, Figure 7$)$, while isolated aerobic exercise training therapy had no impact on cardiac lipid peroxides compared with KOS group (Figure 7).

\section{Discussion}

Here, we report that combined carvedilol and aerobic exercise training therapies integrate the distinctly different beneficial effects of isolated therapies on exercise capacity, ventricular function and remodeling associated with improved $\mathrm{Ca}^{2+}$ homeostasis and reduced ventricular oxidative stress. The main findings of the present study are that combined carvedilol and aerobic exercise training therapies: a) reduce mortality toward WT mice group level, b) improve exercise tolerance, c) re-establish left ventricle contractility and leads to a ventricular reverse remodeling, d) alter expression levels of proteins involved in $\mathrm{Ca}^{2+}$ handling and, e) prevent ventricular lipid peroxidation.

In the present study, we observed that improved exercise tolerance was restricted to exercised groups while re-established ventricular contractility and anti-cardiac remodeling were mainly observed in the groups receiving carvedilol. It is well-known that increased exercise tolerance, improved ventricular function and reduced cardiac remodeling are associated with improved survival in cardiovascular disease [20-22].

Presently, both isolated aerobic exercise training and carvedilol therapies improved survival, which might be related to their specific effects on exercise tolerance (KOT group) and ventricular function/remodeling, respectively. Therefore, our data suggest that aerobic exercise training and $\beta$-blockers have a positive impact on $\mathrm{HF}$ by different mechanisms and these findings corroborate previous studies from our group [9,13-15] and others $[7,23,24]$. Of great interest, here we demonstrated that the positive effect of isolated therapies on survival, exercise tolerance and cardiac contractility and remodeling are preserved and integrated when aerobic exercise training is associated to carvedilol therapy.

Regarding the molecular mechanisms underlying the positive effects of isolated therapies, we have previously provided compelling evidence that aerobic exercise training improves cardiac $\mathrm{Ca}^{2+}$ handling [13,14], which is directly associated with cardiac contractility. In fact, we presently observed that aerobic exercise training improves cardiac $\mathrm{Ca}^{2+}$ homeostasis by increasing 
A

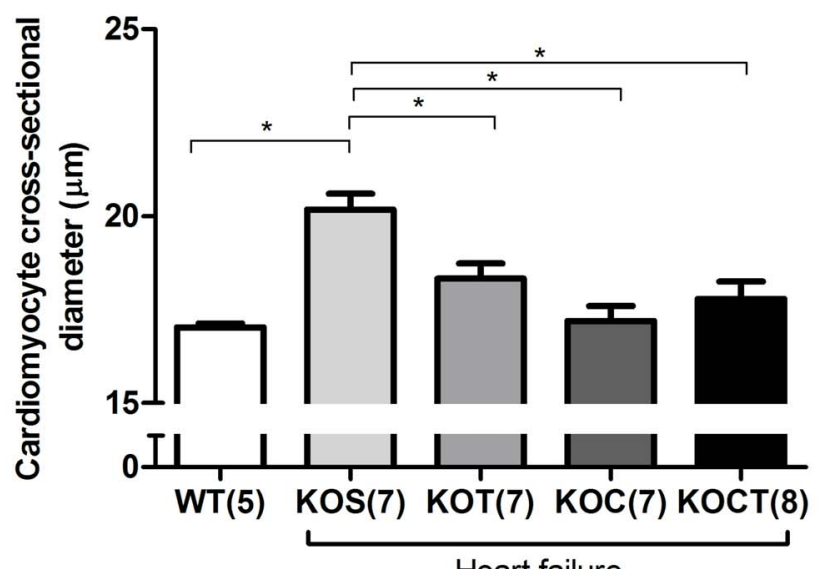

B

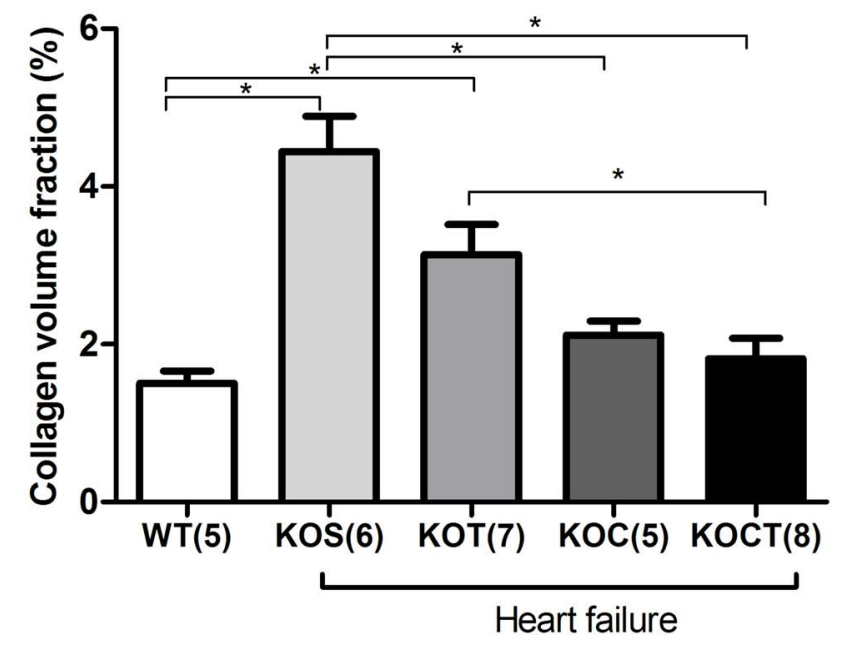

Figure 4. Cardiomyocytes cross-sectional area (A) and ventricular collagen volume fraction (B) in control (WT), heart failure placebo (KOS), heart failure exercise trained (KOT), heart failure carvedilol-treated (KOC) and, heart failure carvediloltreated and exercise trained (KOCT) mice. Data are presented as mean \pm SE. The number of animals studied is indicated by numerals on the abscissa. ${ }^{*} \mathrm{P}<0.05$ among groups indicated by lines.

doi:10.1371/journal.pone.0062452.g004

the peak of $\mathrm{Ca}^{2+}$ transient, decreasing diastolic $\mathrm{Ca}^{2+}$ decay associated with increased phosphorylation of PLN at Ser $^{16}$ and $\mathrm{Thr}^{17}$ in KO mice. Considering that phosphorylation of PLN at either Ser $^{16}$ or $\mathrm{Thr}^{17}$ removes the inhibitory effect of PLN on SERCA2, it is reasonable to suggest that increased levels of phosphorylated PLN induced by aerobic exercise training contribute to the improved $\mathrm{Ca}^{2+}$ reuptake, as reported by faster diastolic $\mathrm{Ca}^{2+}$ decay. Carvedilol treatment had no impact on $\mathrm{Ca}^{2+}$ transient dynamics, since it failed to increase $\mathrm{Ca}^{2+}$ peak and prolonged $\mathrm{Ca}^{2+}$ decay, and significantly attenuated phosphoSer ${ }^{16}$-PLN and Ser ${ }^{17}$-PLN expression levels. We previously demonstrated that despite no impact on $\mathrm{Ca}^{2+}$ transients, carvedilol reduces phosphorylation of troponin I at $\operatorname{Ser}^{23 / 24}$-residues [9], which ultimately leads to an increased sensitivity of contractile myofilament to $\mathrm{Ca}^{2+}$ associated with the antioxidant properties of
A
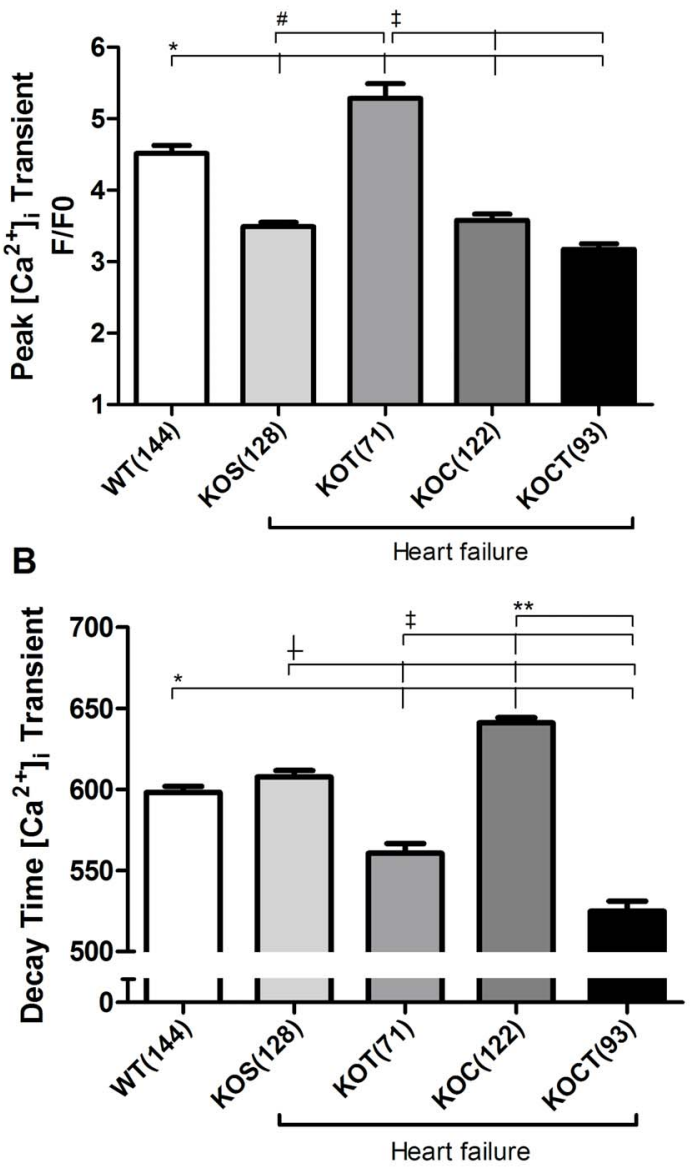

Figure 5. Intracellular $\mathrm{Ca}^{2+}$ transient in isolated ventricular myocytes in control (WT), heart failure placebo (KOS), heart failure exercise trained (KOT), heart failure carvedilol-treated (KOC) and, heart failure carvedilol-treated and exercise trained (KOCT) mice. A: Averaged data showing peak of $\mathrm{Ca}^{2+}$ transient $\mathrm{B}$ : Bar graph shows a comparison of $\mathrm{Ca}^{2+}$ transient kinetics (time from peak to $90 \%$ decay) between the different groups of cells. Data are presented as mean \pm SE. The number of cells studied is indicated by numerals on the abscissa. ${ }^{*} \mathrm{P}<0.05$ for WT vs. indicated groups; (WT vs. all groups in Panel $A$ and WT vs. KOT, KOC and KOCT in Panel B); ${ }^{\#} P<0.05$ for KOS vs. KOT; ${ }^{+} \mathrm{P}<0.05$ for KOS vs. KOT, KOC and KOCT; ${ }^{\ddagger} \mathrm{P}<0.05$ for KOT vs. KOC and $\mathrm{KOCT} ;{ }^{* *} \mathrm{P}<0.05$ for KOC vs. KOCT. doi:10.1371/journal.pone.0062452.g005

carvedilol. In fact, carvedilol has a well-recognized antioxidant activity [25-27], which led us to investigate whether it would reduce myocardial oxidative stress in $\mathrm{KO}$ groups. Indeed, only groups treated with carvedilol reduced ventricular oxidative stress.

The molecular effects of combined carvedilol and aerobic exercise training therapies also rely on the integrative effects of isolated therapies. Combined therapies increased SERCA2 and phosphorylation of PLN expression at both $\mathrm{Ser}^{16}$ and $\mathrm{Thr}^{17}$ residues and reduced myocardial oxidative stress. Interestingly, these responses reflected on faster $\mathrm{Ca}^{2+}$ decay time observed in KOCT compared with KOS, KOT and KOC groups, which suggests a synergistic action of combined therapies on ventricular relaxation. Therefore, we provide compelling evidence that the combination of carvedilol and aerobic exercise training may represent better prognostic power in life long-treatment. It will be important, however, to further explore whether life long-term combination therapy leads to increased efficacy in reducing mortality and if so, what is the potential role of the preferential 
A

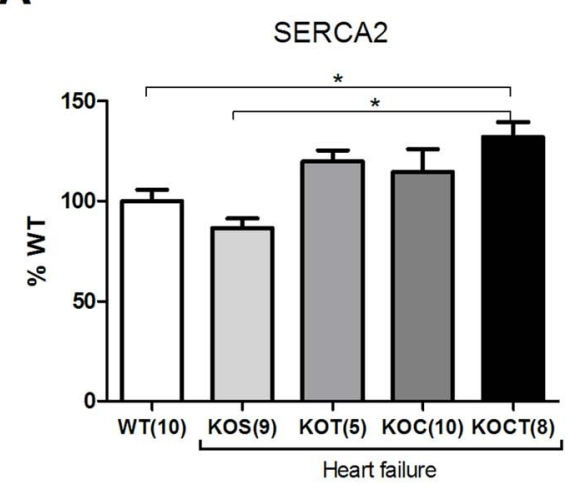

C

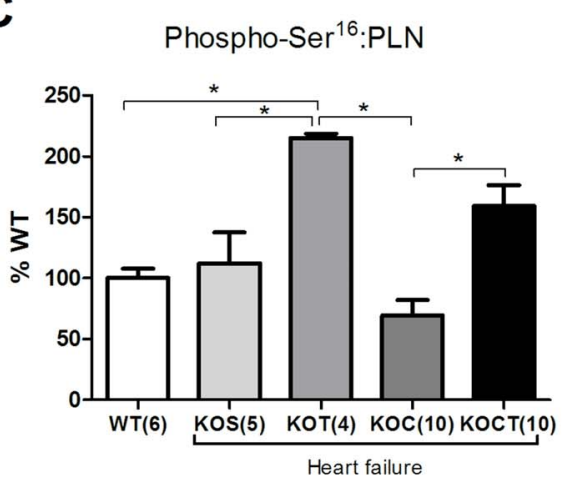

E

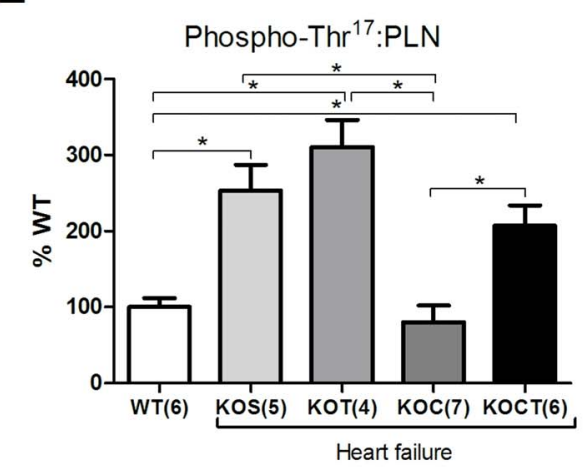

G

RyR

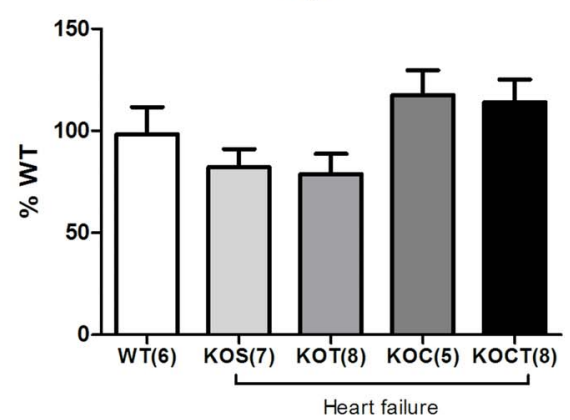

B

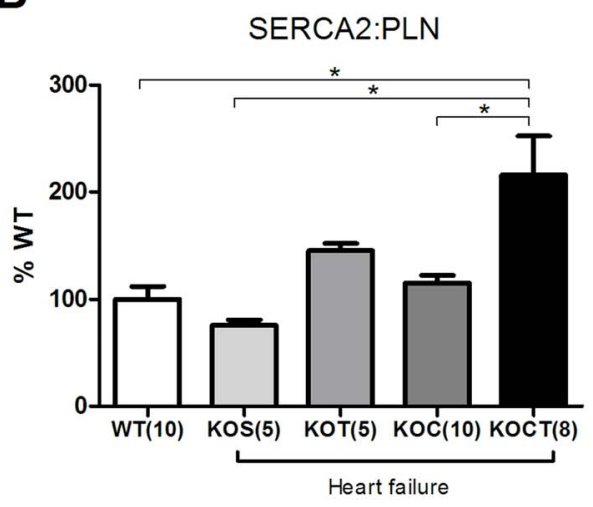

D

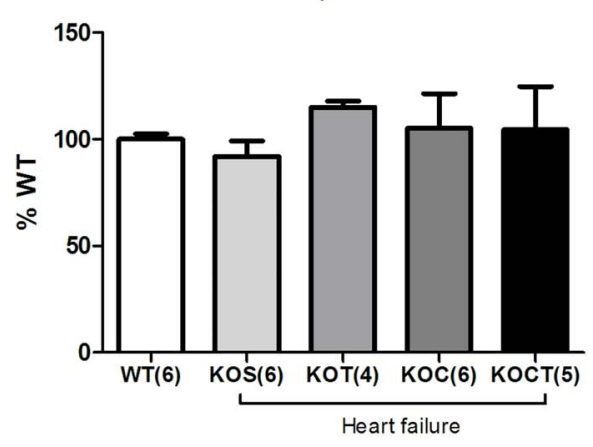

$\mathbf{F}$

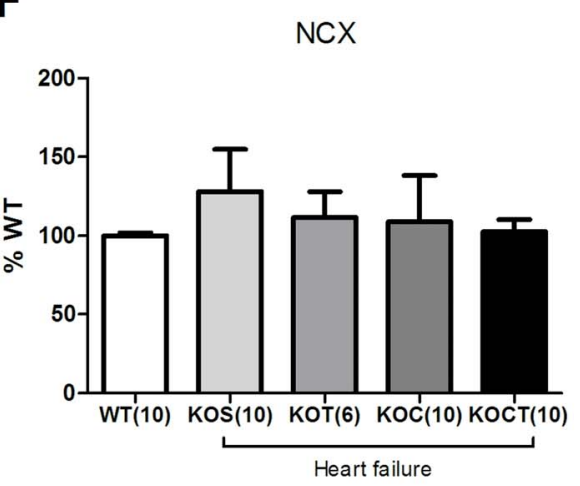

$\mathrm{H}$

PLN

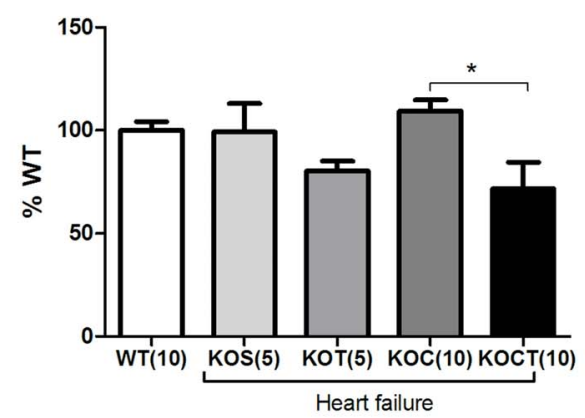

Figure 6. SERCA2 (A), SERCA2A:PLN (B), Phospho-Ser ${ }^{16}-$ PLN:PLN (C), Phosphatase 1 (D), Phospho- Thr ${ }^{17}-$ PLN:PLN (E), Na ${ }^{+}-C^{2+}$ exchanger (NCX, F), Ryanodine receptor (RyR, G), Phospholamban (PLN, H). Data are presented as mean \pm SE. ${ }^{*} \mathrm{P}<0.05$ among groups indicated by lines.

doi:10.1371/journal.pone.0062452.g006 


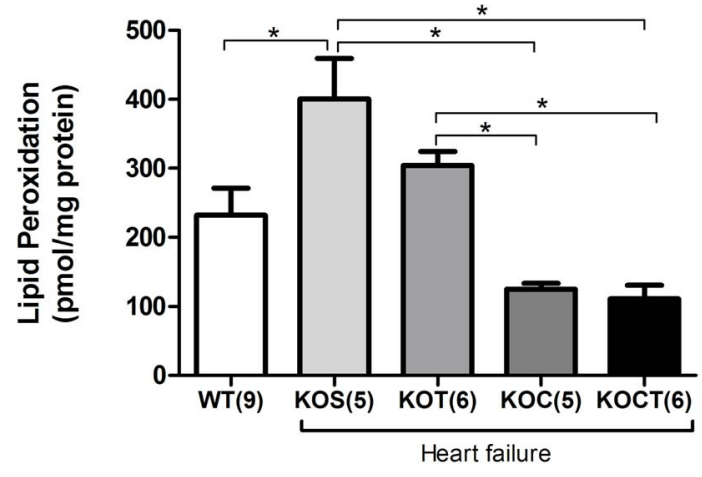

Figure 7. Lipid peroxidation in control (WT), heart failure placebo (KOS), heart failure exercise trained (KOT), heart failure carvedilol-treated (KOC) and, heart failure carvediloltreated and exercise trained (KOCT) mice. Data are presented as mean \pm SE. The number of animals studied is indicated by numerals on the abscissa. ${ }^{*} \mathrm{P}<0.05$ among groups indicated by lines. doi:10.1371/journal.pone.0062452.g007

$\mathrm{Ca}^{2+}$ transient improvement associated to aerobic exercise training versus the greater ventricular anti-remodeling and antioxidant effects mediated by carvedilol.

The relative contribution of aerobic exercise training and carvedilol for improving cardiac function and survival is hampered by known limitations in clinical studies including patients number,

\section{References}

1. Adamopoulos S, Parissis JT, Kremastinos DT (2003) New aspects for the role of physical training in the management of patients with chronic heart failure. Int J Cardiol 90: 1-14.

2. Grassi G, Seravalle G, Cattaneo BM, Lanfranchi A, Vailati S, et al. (1995) Sympathetic activation and loss of reflex sympathetic control in mild congestive heart failure. Circulation 92: 3206-3211.

3. Zucker IH, Patel KP, Schultz HD (2012) Neurohumoral stimulation. Heart Fail Clin 8: 87-99.

4. Barretto AC, Santos AC, Munhoz R, Rondon MU, Franco FG, et al. (2009) Increased muscle sympathetic nerve activity predicts mortality in heart failure patients. Int J Cardiol 135: 302-307.

5. Burger AJ, Aronson D (2001) Activity of the neurohormonal system and its relationship to autonomic abnormalities in decompensated heart failure. J Card Fail 7: 122-128.

6. Mahon NG, Young JB, McKenna WJ (2002) Beta adrenergic blockers in chronic congestive cardiac failure: a call for action. Eur J Intern Med 13: 96100 .

7. De Matos LD, Gardenghi G, Rondon MU, Soufen HN, Tirone AP, et al. (2004) Impact of 6 months of therapy with carvedilol on muscle sympathetic nerve activity in heart failure patients. J Card Fail 10: 496-502.

8. Khattar RS (2003) Effects of ACE-inhibitors and beta-blockers on left ventricular remodeling in chronic heart failure. Minerva Cardioangiol 51: $143-154$.

9. Bartholomeu JB, Vanzelli AS, Rolim NP, Ferreira JC, Bechara LR, et al. (2008) Intracellular mechanisms of specific beta-adrenoceptor antagonists involved in improved cardiac function and survival in a genetic model of heart failure. J Mol Cell Cardiol 45: 240-249.

10. Vanzelli AS, Medeiros A, Sirvente Rde A, Salemi VM, Mady C, et al. (2010) Association of physical training with beta-blockers in heart failure in mice. Arq Bras Cardiol 95: 373-380.

11. Chung CJ, Schulze PC (2011) Exercise as a nonpharmacologic intervention in patients with heart failure. Phys Sportsmed 39: 37-43.

12. Brum PC, Bacurau AV, Medeiros A, Ferreira JC, Vanzelli AS, et al. (2011) Aerobic exercise training in heart failure: impact on sympathetic hyperactivity and cardiac and skeletal muscle function. Braz J Med Biol Res 44: 827-835.

13. Medeiros A, Rolim NP, Oliveira RS, Rosa KT, Mattos KC, et al. (2008) Exercise training delays cardiac dysfunction and prevents calcium handling abnormalities in sympathetic hyperactivity-induced heart failure mice. J Appl Physiol 104: 103-109.

14. Rolim NP, Medeiros A, Rosa KT, Mattos KC, Irigoyen MC, et al. (2007) Exercise training improves the net balance of cardiac $\mathrm{Ca} 2+$ handling protein expression in heart failure. Physiol Genomics 29: 246-252. etiology, co-morbidities and genetic makeup to name a few. In the present study, we took advantage of a congenic genetic model of sympathetic hyperactivity-induced HF to assess potential differences in cardiac structure and function when the animals were treated with carvedilol or exercised at a moderate level to achieve comparable HR exhibited by the WT group for two months. Therefore, the isolated effect of each therapy could be considered equally efficient in reducing HR, an important marker of both aerobic exercise training and $\beta$-blocker therapy efficacy.

\section{Conclusion}

Taken together, we provided evidence that combined therapies with carvedilol and aerobic exercise training integrate the beneficial effects of isolated ones on survival, exercise tolerance and cardiac contractility and structure. The molecular mechanisms underlying the beneficial effects of combined therapies rely on the improved cardiac $\mathrm{Ca}^{2+}$ homeostasis mainly related to moderate aerobic exercise training effect and reduced myocardial oxidative stress and reverse ventricular remodeling associated with carvedilol therapy.

\section{Author Contributions}

Conceived and designed the experiments: ASV PCB. Performed the experiments: ASV AM NLR JBB TFC LGB KCM RS ERG. Analyzed the data: ASV NLR KCM. Contributed reagents/materials/analysis tools: VS CM SG CEN PCB. Wrote the paper: ASV AM NLR PCB.

15. Medeiros A, Vanzelli AS, Rosa KT, Irigoyen MC, Brum PC (2008) Effect of exercise training and carvedilol treatment on cardiac function and structure in mice with sympathetic hyperactivity-induced heart failure. Braz J Med Biol Res 41: 812-817.

16. Brum PC, Kosek J, Patterson A, Bernstein D, Kobilka B (2002) Abnormal cardiac function associated with sympathetic nervous system hyperactivity in mice. Am J Physiol Heart Circ Physiol 283: H1838-1845.

17. Johns C, Gavras I, Handy DE, Salomao A, Gavras H (1996) Models of experimental hypertension in mice. Hypertension 28: 1064-1069.

18. Guatimosim S, Sobie EA, dos Santos Cruz J, Martin LA, Lederer WJ (2001) Molecular identification of a TTX-sensitive $\mathrm{Ca}(2+)$ current. Am J Physiol Cell Physiol 280: C1327-1339.

19. Nourooz-Zadeh J, Tajaddini-Sarmadi J, Wolff SP (1994) Measurement of plasma hydroperoxide concentrations by the ferrous oxidation-xylenol orange assay in conjunction with triphenylphosphine. Anal Biochem 220: 403-409.

20. Cohn JN, Rector TS (1988) Prognosis of congestive heart failure and predictors of mortality. Am J Cardiol 62: 25A-30A.

21. Likoff MJ, Chandler SL, Kay HR (1987) Clinical determinants of mortality in chronic congestive heart failure secondary to idiopathic dilated or to ischemic cardiomyopathy. Am J Cardiol 59: 634-638.

22. Myers J, Prakash M, Froelicher V, Do D, Partington S, et al. (2002) Exercise capacity and mortality among men referred for exercise testing. N Engl J Med 346: 793-801.

23. Flesch M, Maack C, Cremers B, Baumer AT, Sudkamp M, et al. (1999) Effect of beta-blockers on free radical-induced cardiac contractile dysfunction. Circulation 100: 346-353.

24. Fraga R, Franco FG, Roveda F, de Matos LN, Braga AM, et al. (2007) Exercise training reduces sympathetic nerve activity in heart failure patients treated with carvedilol. Eur J Heart Fail 9: 630-636.

25. Koitabashi N, Arai M, Tomaru K, Takizawa T, Watanabe A, et al. (2005) Carvedilol effectively blocks oxidative stress-mediated downregulation of sarcoplasmic reticulum Ca2+-ATPase 2 gene transcription through modification of Sp1 binding. Biochem Biophys Res Commun 328: 116-124.

26. Nakahira A, Minamiyama Y, Takemura S, Hirai H, Sasaki Y, et al. (2010) Coadministration of carvedilol attenuates nitrate tolerance by preventing cytochrome p450 depletion. Circ J 74: 1711-1717.

27. Zhuang XF, Yin CQ, Wang HY, Sun NL (2009) Distinctive effects of carvedilol in the non-infarct zone: remodelling of the ligated rat heart linked to oxidative stress. J Int Med Res 37: 1354-1364. 